\title{
Modeling of conjugated heat and mass transfer in the entrance region of falling liquid film at various values of the Froude number
}

\author{
Maria V. Bartashevich, ${ }^{1, *}$ \\ ${ }^{1}$ Kutateladze Institute of Thermophysics, 630090 Novosibirsk, Russia
}

\begin{abstract}
Mathematical model of conjugated heat and mass transfer in absorption on the entrance region of the semi-infinite liquid film of lithium bromide water solution is investigated for different values of Froude number. The calculations shown that larger values of Froude number corresponds to a smaller thickness of the falling film. It was demonstrated that for large values of the Froude number the heat transfer from the surface is greater than for smaller values.
\end{abstract}

\section{Problem statement}

In absorption chillers and in the spray-type devices the heat and mass transfer processes take place and proceed with a pressure-driven liquid film flow through holes or slits. In other types of devices, including film-like devices, such flows are possible at the exit of the distributors. The liquid flow with a free boundaries in the real devices are often conjugated by the various heat and mass transfer processes such as absorption.

The solutions for simple problems of the coupled heat and mass transfer during absorption at the liquid/gas interfaces of different geometry types (films, jets, drops, sheets) are given in $[1,2]$. Modelling of the non-isothermal absorption of vapour into expanding liquid sheets was performed by Acosta-Iborra and co-workers [3]. In [4] it has been shown that a significant change of the salt concentration leads to different absorption regimes.

In present work we have been investigated the coupled heat and mass transfer during absorption in the entrance region of the falling axisymmetric semi-infinite film surrounded by the pure vapour or gas at various values of the Froude number.

We assume, that the liquid film falls under the action of gravity with the uniform vertical velocity $u_{i}$. Downstream, the velocity is defined as $u=\sqrt{u_{i}^{2}+2 g x}$. The initial thickness of the film is $h_{i}$. The Froude number $F r=\left(g h_{i}\right) / u_{i}^{2}$.

The process of heat and mass transfer at film absorption is described by the equations of heat conductivity and diffusion:

$$
u \partial T / \partial x=a \partial^{2} T / \partial y^{2}
$$

\footnotetext{
* Corresponding author: bartashevichmv@gmail.com
} 


$$
u \partial C / \partial x=D \partial^{2} C / \partial y^{2}
$$

$a$ is thermal diffusivity, $\mathrm{m}^{2} / \mathrm{s} ; D$ is diffusion coefficient, $\mathrm{m}^{2} / \mathrm{s}$. The boundary conditions at the inlet at $x=0$ are:

$$
T=T_{i}, C=C_{i}, h=h_{i} .
$$

Similarly to [1], the equilibrium of solution-vapor system occurs on the interface at $y=h$ :

$$
C_{d}=f\left(T_{d}\right), \lambda \partial T / \partial y=\chi \partial C / \partial y
$$

where $\chi=L \rho D /\left(1-C_{i}\right), L$ is latent heat of phase transformation, $\mathrm{J} / \mathrm{kg} ; \rho$ is solution density, $\mathrm{kg} / \mathrm{m}^{3} ; \lambda$ is heat conductivity, $\mathrm{W} /(\mathrm{mK})$. This equilibrium condition joins equilibrium temperature $T_{d}$ with concentration $C_{d}$. We assume the asymptotic boundary condition at $y^{\prime} \rightarrow \infty:$

$$
T=T_{i}, C=C_{i},
$$

where $y^{\prime}=y-h$ and directed from the interface to the film depth. The conditions of absorption are $T_{i}<T_{s}$ and $C_{i}<C_{s}$. Here, $C_{s}=f\left(T_{i}\right)$ is equilibrium concentration, corresponding to initial temperature of solution $T_{i} ; T_{s}$ is equilibrium temperature corresponding to initial concentration $C_{i}\left(C_{i}=f\left(T_{s}\right)\right)$, similarly to [1]. We have the temperature gradient at the free liquid solution interface:

$$
\frac{\partial T}{\partial y}=\Delta T T_{i}\left(F r^{1 / 2} P e^{1 / 2} \frac{1}{h_{i} \sqrt{\pi}\left(2 F r \frac{x}{h_{i}}+1\right)^{1 / 4}}\right),
$$

where $\Delta T=T_{s}-T_{i}$ is temperature difference between initial $T_{i}$ and equilibrium $T_{s}$ temperatures, $P e=\frac{u_{i} h_{i}}{a}$ is a Peclet number. Also, Nusselt number dependence from longitudinal coordinate can be obtained as:

$$
N u(x)=T_{i} F r^{1 / 2} P e^{1 / 2} \frac{1}{\sqrt{\pi}\left(2 F r \frac{x}{h_{i}}+1\right)^{1 / 4}} .
$$

\section{Calculations}

Numerical calculations were carried out for different values of Froude number. The results of calculations of absorption on the entrance region of the semi-infinite liquid film of lithium bromide water solution are given in Fig.1 - Fig.2. The calculations were performed at $T_{i}=20^{\circ} \mathrm{C}, T_{s}=46{ }^{\circ} \mathrm{C}, C_{i}=0.4, C_{s}=0.56, F r=0.25,0.5,1.0, P e=200,400$. 




Fig. 1. The Nusselt number along the film-flow direction at the entrance region for different values of Froude number, $P e=200.1-F r=0.25 ; 2-F r=0.5 ; 3-F r=1.0$.

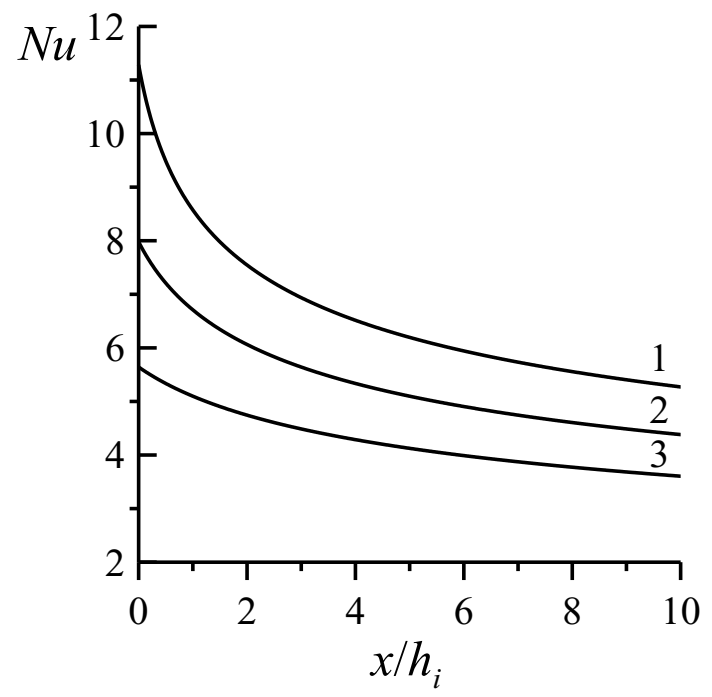

Fig. 2. The Nusselt number along the film-flow direction at the entrance region for different values of Froude number, $P e=400.1-F r=0.25 ; 2-F r=0.5 ; 3-F r=1.0$.

Fig. 1 and Fig. 2 show the calculated distributions of the Nusselt number along the filmflow direction for different values of the Froude and Peclet numbers. For $P e=400$ the Nusselt number is almost 1.5 times more than for $P e=200$ at the inlet. However, with the distance from the entrance region, the difference decreases.

\section{Conclusions}

In the absorption heat pumps, four heat and mass transfer processes can occur simultaneously: absorption, desorption, condensation, evaporation. In this paper, the Nusselt number $(\mathrm{Nu})$, for different values of the Froude $(\mathrm{Fr})$ and Peclet $(\mathrm{Pe})$ numbers, was 
calculated for the absorption element of the heat pump in the entrance region of the freeflowing film. The dependence of the Nusselt number on the dimensionless longitudinal coordinate is constructed. It was shown in [2] that a larger thickness of the Froude number corresponds to a smaller thickness of the flowing film. In the calculations presented here it was demonstrated that for the large values of the Froude number, the heat transfer from the surface is greater than for smaller values and the heat transfer more intensive for large Peclet numbers for such regime of the falling film.

This work was carried out at the Kutateladze Institute of Thermophysics SB RAS and financially supported by the Russian Science Foundation (project number 15-19-10025).

\section{References}

1. V.E. Nakoryakov, N.I. Grigorieva, Non-Isothermal Absorption in Thermal Transformers (Nauka, Novosibirsk, 2010)

2. V.E. Nakoryakov, N.I. Grigoryeva, M.V. Bartashevich, Int. J. Heat Mass Transfer 54 (21-22) (2011)

3. A. Acosta-Iborra, N. Garcia-Hernando and D. Santana, Int. J. Heat Mass Transfer 52 (2009)

4. V.E. Nakoryakov, S.Y. Misyura, J. Eng. Thermophys. 25 (2016) 